\title{
Methane clathrate hydrate infrared spectrum
}

\section{Near-infrared overtones, combination modes and cages assignments}

\author{
E. Dartois, ${ }^{\star}$, D. Deboffle, and M. Bouzit
}

\begin{abstract}
Institut d'Astrophysique Spatiale, UMR-8617, Université Paris-Sud, Bât. 121, 91405 Orsay, France
\end{abstract}
e-mail: emmanuel.dartois@ias.u-psud.fr

Received 11 November 2009 / Accepted 28 January 2010

\begin{abstract}
Context. Recently, we recorded the infrared spectrum of the methane clathrate hydrate stretching mode at low temperature, a caged compound of possible interest for solar system studies as well as interstellar ice mantles.

Aims. We provide a practical infrared spectroscopic identification for methane clathrate hydrate to examine its astrophysical presence or absence. We investigate the crystal field induced shifts, and assign the different transitions to the different encaged molecules environments in this clathrate hydrate.

Methods. A methane clathrate crystal is produced in an infrared transmitting moderate-pressure closed cell. Using Fourier transform infrared (FTIR) spectroscopy, the overtones $\left(3 v_{4}, 2 v_{3}\right)$ and combination modes $\left(v_{2}+2 v_{4}, v_{1}+v_{4}, v_{3}+v_{4}, v_{2}+v_{3}, v_{3}+2 v_{4}, 2 v_{2}+2 v_{4}, v_{2}+\right.$ $\left.v_{3}+v_{4}\right)$ falling in the $6000-3000 \mathrm{~cm}^{-1}(\sim 1.65-3.4 \mu \mathrm{m})$ and their temperature behaviour are investigated. In addition, non-astrophysical $\mathrm{CH}_{4} / \mathrm{CF}_{4}$ gas mixtures are used to build clathrates with different methane large and small cage occupancies to help in assignments. Results. Combination modes show the two distinct cages and the quasi-free rotor low temperature ro-vibrational structure expected for methane clathrate hydrates. A comparison with the pure phase I is performed. Implications for methane clathrate hydrate detection are clearly identified.

Conclusions. Solid methane actual remote observations of solar system objects surfaces do not display the clathrate hydrates' specific shift and occupancy signatures. Observationnally, a search for their infrared spectroscopic specific signatures should be performed, focusing on thermodynamically favourable objects like trans- neptunian objects (TNOs) or recently exposed (e.g. fresh impact) planet (or their satellites) surfaces. On the modeling side, efforts must be undertaken to progressively implement clathrate formation kinetics.
\end{abstract}

Key words. line: identification - comets: general - planets ans satellites: general - ISM: lines and bands - dust, extinction - molecular data

\section{Introduction}

The existence of clathrate hydrate dates back to the early 19th century, when Faraday (1823) defined the chlorine clathrate hydrate discovered by Davy in 1810. Since then, a huge amount of effort has been made to study clathrate hydrates not only for the intrinsinc scientific interest in this new phase, but also because they were shown in the last century to be responsible for plugs in pipelines, forming ice stoppers endangering gas and oil field explotations as well as the life of the workers. Methane clathrate hydrate provides a (although difficult to exploit) future energy resource in addition to petroleum, as it is an important constituent of the permafrost. Clathrate hydrates could also provide an ecologically clean storage medium for small molecules, and in particular for safe hydrocarbons or hydrogen transportation or for engine applications. Their properties can also be used to trigger phase separation or purification for chemical constituents in mixed phases, and are promising to sequestrate greenhouse gases like $\mathrm{CO}_{2}$ (Chatti et al. 2005, and references therein).

Clathrate hydrate thus enters into diverse applications, and space astrophysics is no exception to the rule, as they were early proposed to explain the anomalous desorption behaviour of

* Part of the equipment used in this work has been financed by the French INSU-CNRS program "Physique et Chimie du Milieu Interstellaire" (PCMI). gaseous parent molecules in comets (e.g. Delsemme \& Swings 1952), by providing a dissociation pressure curve intermediate between the pure compound sublimation equilibrium and the water ice phase. It was subsequently applied to understand other objects of the solar system, from planets to their moons and outer solar nebula planetesimals (e.g. Lunine \& Stevenson1985; Sill \& Wilkening 1978; Miller 1961). Nowdays it is the source for many astrophysical investigations with application ranging from explaining part of giant planets (and/or their satellites), observed molecular abundances (e.g. Hersant et al. 2004), Mars carbon dioxide clathrate hydrates (e.g. Schmitt et al. 2003; Kuhs \& Klapproth 2000), or ethane sequestration on Titan (Mousis \& Schmitt 2008). We experimentally investigate the near to midinfrared spectroscopic signature of type I methane clathrate hydrate, as well as $\mathrm{CH}_{4} / \mathrm{CF}_{4}$ clathrate mixtures to decipher the different cage assignments. We describe the experiments in Sect. 2 and the associated results in Sect. 3. We discuss in Sect. 4 the spectra and cage identification and Sect. 5 discuss the astrophysical implications for clathrate hydrate observations. We then conclude.

\section{Experiments}

A new evacuable enclosed cell was built to supersede the cell used in our previous clathrate hydrate experimental setup (Dartois \& Deboffle 2008). The two thick (4 mm) lateral ZnSe 


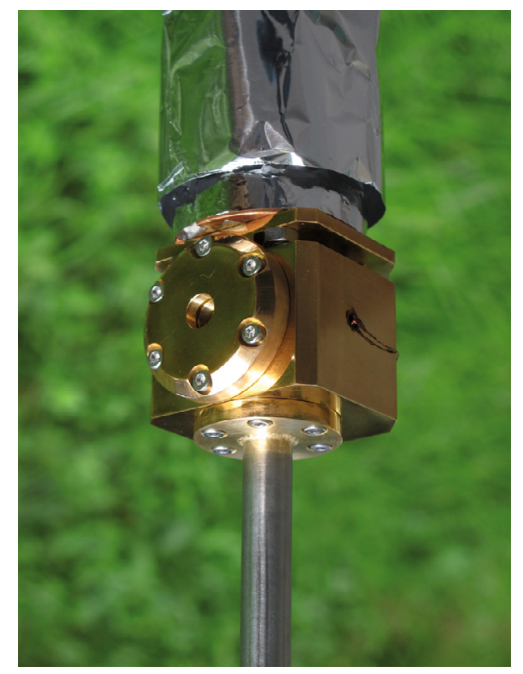

Fig. 1. Low temperature moderate pressure closed cell used to nucleate and characterise the methane clathrate hydrate. The top is attached to a liquid $\mathrm{He}$ transfer cold finger, masked by a thermal shield cylinder. The lower injection tube is used both to inject gaseous water and methane as well as to evacuate it once the hydrate is formed. The two cylindrical gold coated copper part support the ZnSe windows, offering a $3.5 \mathrm{~mm}$ radius aperture to the infrared beam.

or Sapphire windows diameter were reduced to $13 \mathrm{~mm}$ diameter and were mounted so that the high pressure acting inside improved their sealing by squeezing their indium gasket. In addition, the copper cell was optically polished and subsequently gold-coated by electrolysis to improve its reflectivity in the infrared, ensuring a lower heat load. The cell was thermally coupled to a liquid He transfer cold finger, placed in a high vacuumevacuated cryostat $\left(P<10^{-7}\right.$ mbar $)$. A stainless-steel injection tube for the entrance or evacuation of the gases was sealed at the bottom of the cell and ended with a four port injector inside. An image of the cell is shown in Fig. 1.

The formation of the methane hydrate follows a procedure described previously (Dartois \& Deboffle 2008), in which the ice film is formed first and pressurized with methane gas during approximately two days in a region of the $(P, T)$ diagram above the equilibrium curve for methane clathrate hydrate. Then the temperature is decreased while the excess methane is progressively evacuated and care is taken to maintain the system below the vaporization or sublimation of the methane phase diagram. Methane clathrate hydrate near-infrared FTIR spectra were recorded with a Bruker IFS $66 \mathrm{v}$ at a resolution of $0.5 \mathrm{~cm}^{-1}$ and 3 terms Blackman-Harris apodization, with a globar IR source, $\mathrm{KBr}$ beamsplitter and an $\mathrm{HgCdTe}$ detector cooled at $\mathrm{LN}_{2}$. The stretching mode region spectrum is the one already discussed in Dartois \& Deboffle (2008). Pure methane spectra were recorded in its two phases I (25K) and II (5.6K) in the same cell for comparison.

Mixed methane-carbon tetrafluoride clathrate hydrates were produced by maintaining the cell above the equilibrium pressure curve measured by Mooijer-van den Heuvel et al. (2006). Experiments with $\mathrm{CH}_{4} / \mathrm{CF}_{4}$ gas molar ratios of $5 \%, 15 \%$ and $50 \%$, provided by Messer special gases (with an uncertainty of $2 \%$ relative to the methane content) were injected into the cell. $\mathrm{CF}_{4}$ is not astrophysically relevant in such high proportions. The aim of these mixtures is to modify cage occupancies in the clathrate, once formed, as will be discussed in the results. The set of experiments were conducted over more than six months, and the cell thermal shield insulation was progressively improved

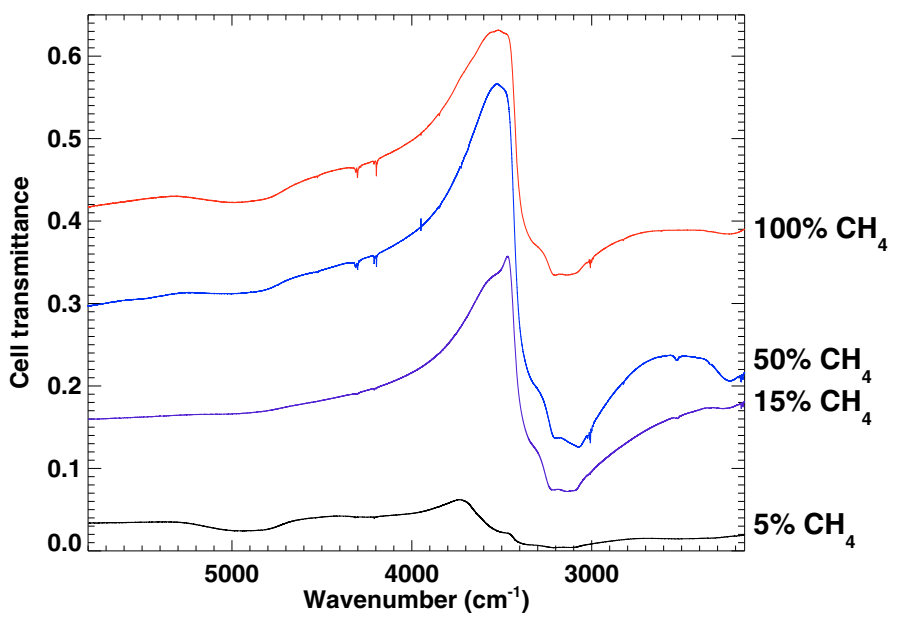

Fig. 2. Typical raw cell transmittance deformed spectra containing the clathrate crystallites for different $\mathrm{CH}_{4} / \mathrm{CF}_{4}$ used in this study, at low temperatures.

with respect to the initial methane hydrate experiments. Thus the lowest temperatures reached varied from $7 \mathrm{~K}$ for the methane clathrate stretching mode (Dartois \& Deboffle 2008) to as low as $\sim 5.05 \mathrm{~K}$ for the $\mathrm{CH}_{4} / \mathrm{CF}_{4} 50 \%$ molar ratio. The resolution for these FTIR spectra also varied and was set to $0.4 \mathrm{~cm}^{-1}$ and three terms Blackman-Harris apodization for the 5\% and $15 \%$ mixtures and $0.15 \mathrm{~cm}^{-1}$ and four points apodization for the $50 \%$ mixture, respectively. They were recorded in different source configurations (either globar IR source or tungsten lamp), $\mathrm{CaF}_{2}$ or $\mathrm{KBr}$ beamsplitter and an InSb detector cooled at $\mathrm{LN}_{2}$ to optimise the signal-to-noise in specific wavelength regions. A summary of the $(P, T)$ formation conditions is given in Table 3 in the discussion.

The clathrate ice films are sometimes of poor optical quality (i.e. not planar) as the growth of uncontrolled large clathrate crystallites in the cell deform the FTIR-probed strong water ice bands, as already shown in Fig. 2 of Dartois \& Deboffle (2008) and in Fig. 2 here. The raw clathrate hydrate spectra are dominated by broad water-ice vibrational transitions, the thin lines superimposed on the water-ice features in the NIR pertaining to encaged methane related transitions. The ice deposit is grainy, and additional non-uniformity during the clathrate growth induces some scattering, giving rise to the deformed ice profile observed. This cell allows an efficient growth of clathrates, but a cell to circumvent the uncontrolled defects is being built.

The spectra presented are thus baseline corrected. These growing conditions prevent us from establishing absolute optical constants values, although an estimate of the near-IR relative methane transitions is possible.

\section{Results}

\subsection{Methane clathrate hydrate in the $3500-6000 \mathrm{~cm}^{-1}$ range}

The $\mathrm{CH}_{4}$ clathrate hydrate baseline-corrected near-infrared spectrum covering the $3 v_{4}, v_{1}+v_{4}, v_{3}+v_{4}, v_{2}+v_{3}$ modes is shown in Figs. 3-5. We also observed at low signal-to-noise the $v_{3}+2 v_{4}$, $v_{2}+v_{3}+v_{4}$ and $2 v_{3}$ combination mode range, displayed in Fig. 6 at the lowest temperature for evident line contrast maximization. The spectrum is compared to the phase I and II pure methane spectra recorded in our cell at $5.6 \mathrm{~K}$ and $25 \mathrm{~K}$ respectively, as 
E. Dartois et al.: ethane clathrate hydrate infrared spectrum. II

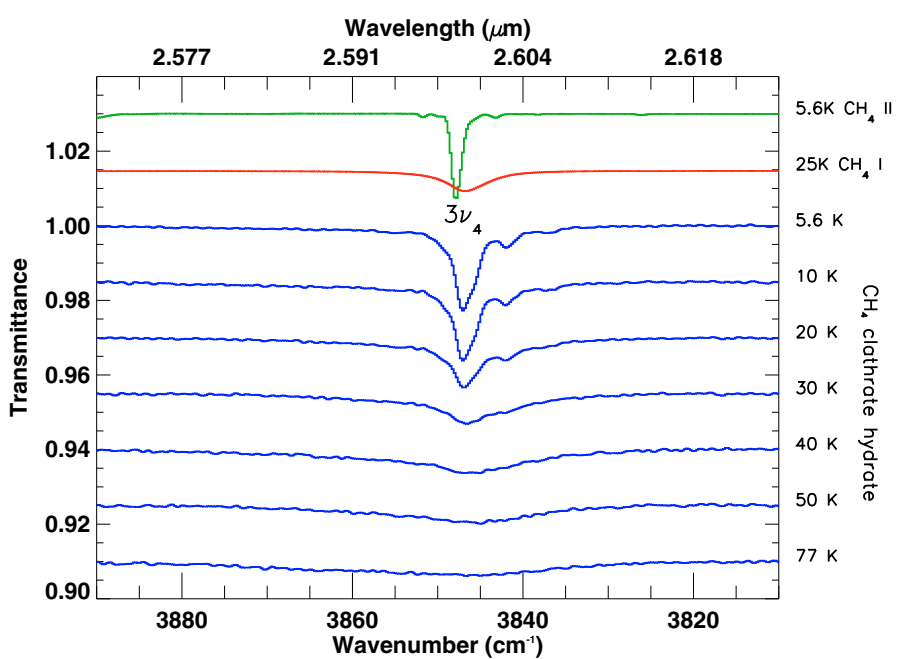

Fig. 3. Temperature-dependent spectrum for methane clathrate hydrate in the $3 v_{4}$ combination-mode absorption range. For comparison, low temperature methane phase II crystalline spectrum at $5.6 \mathrm{~K}$ and phase I at $25 \mathrm{~K}$ recorded using the same cell are shown. The lower set of spectra is the temperature sequence of methane clathrate hydrate spectra, recorded from $5.6 \mathrm{~K}$ to $77 \mathrm{~K}$.

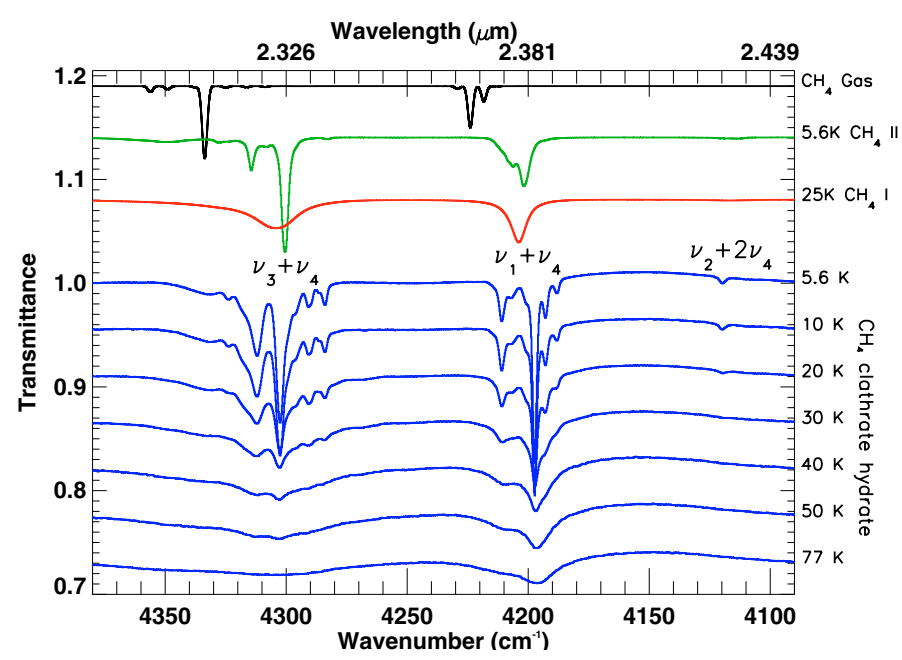

Fig. 4. Temperature-dependent spectrum for methane clathrate hydrate in the $v_{2}+2 v_{4}, v_{1}+v_{4}$ and $v_{3}+v_{4}$ combination-mode absorption range. A comparison to a $5.6 \mathrm{~K}$ gas phase simulated spectrum is shown on top The low temperature methane phase II crystalline spectrum at $5.6 \mathrm{~K}$ and phase I at $25 \mathrm{~K}$ are recorded with the same cell and are shown for comparison. The lower set of spectra is the temperature sequence of methane clathrate hydrate spectra, recorded from $5.6 \mathrm{~K}$ to $77 \mathrm{~K}$.

the transition from phase II to I occurs at $20.53 \mathrm{~K}$ (Vogt \& Pitzer 1976). Some structures appear in the phase II spectrum because some methane molecules are able to rotate in this phase. The observed near-infrared and clathrate hydrate lines (and methane phase II) are given in Table 1. The relative integrated intensities of the measured near-infrared transitions, normalised to the $v_{3}+v_{4}$ vibration mode, are given in Table 2 and are compared to methane-ice values from the literature.

\subsection{Tetrafluoromethane-methane mixed clathrate hydrates}

When tetrafluoromethane-methane is used to form a mixed clathrate hydrate, the larger Van der Waals radius of the $\mathrm{CF}_{4}$ molecule can force the system to adopt structure I with a preferential occupancy of small (S) cages by methane and large (L)

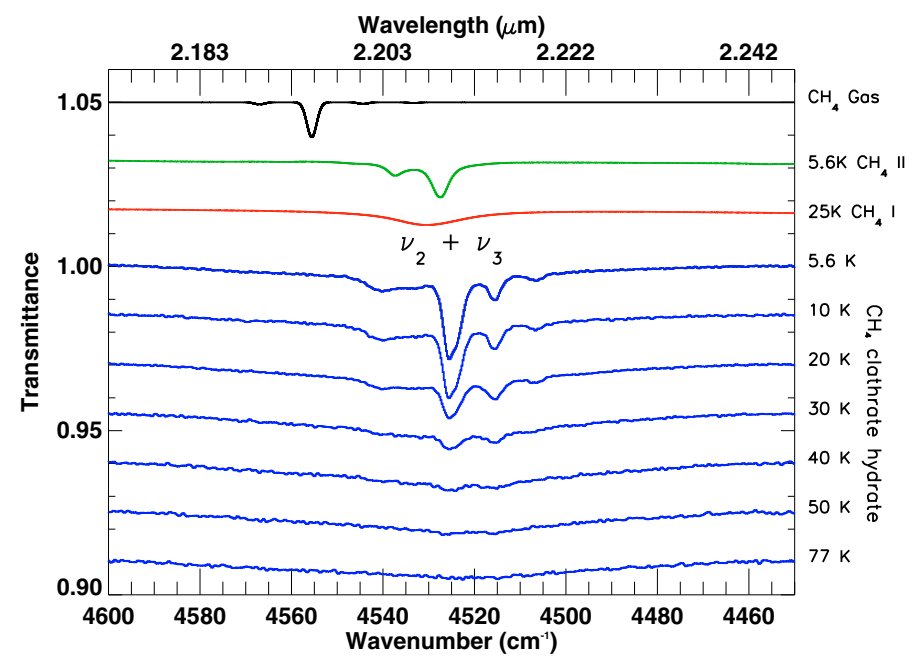

Fig. 5. Temperature dependent spectrum for methane clathrate hydrate in the $v_{2}+v_{3}$ combination mode absorption range. The same set of spectra as in Fig. 3.

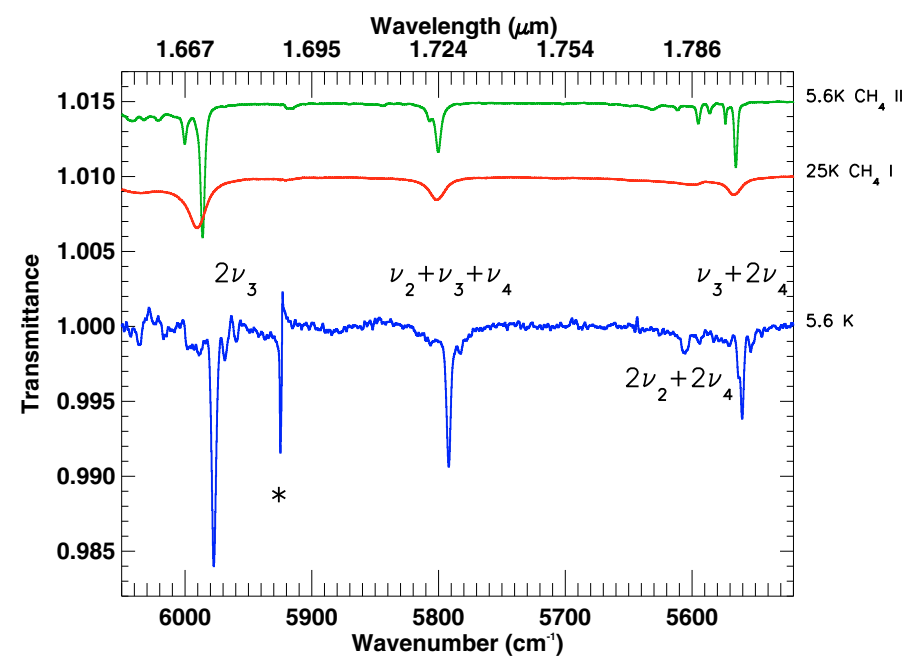

Fig. 6. Lowest temperature spectrum for weak methane clathrate hydrate transitions in the $v_{3}+2 v_{4}, v_{2}+v_{3}+v_{4}$ and $2 v_{3}$ combination mode absorption range.

cages by tetrafluoromethane, as shown in Kunita et al. (2007) with Raman spectroscopy at $291.1 \mathrm{~K}$ for gas $\mathrm{CH}_{4} / \mathrm{CF}_{4}$ molar ratios below $30 \%$ (in the $30-80 \%$ ratios, a structure II clathrate hydrate was formed under Kunita's experimental conditions). As a consequence, the methane $\mathrm{CH}$-stretching mode region should show large cages to small cages occupancy variations. We chose three distinct $\mathrm{CH}_{4}: \mathrm{CF}_{4}$ molar gas ratios $(5 \%, 15 \%$ and $50 \%)$ to perform experiments probing the varying $\mathrm{L} / \mathrm{S}$ occupancies. Following the analysis by Kunita et al. (2007), at higher temperature $(291.1 \mathrm{~K})$ one can expect clathrate $\mathrm{CH}_{4}$ large to small cage occupancy variations. The spectra for the three mixed clathrates and pure $\mathrm{CH}_{4}$ clathrate in the $v_{3}$ antisymmetric stretching mode at low temperature are displayed in Fig. 7 and the first combinations in Fig. 8.

\section{Discussion}

\subsection{Cages identification}

The overlap of large cage $\left(M=5^{12} 6^{2}\right)$ and small cage $\left(S=5^{12}\right)$ methane rovibrational patterns recorded previously prevented us 
Table 1. Observed NIR methane phase II and clathrate hydrate lines.

\begin{tabular}{|c|c|c|}
\hline Dominant transitions & $\begin{array}{c}\mathrm{CH}_{4} \text { II }(5.6 \mathrm{~K})^{a} \\
\bar{v}\left(\mathrm{~cm}^{-1}\right)^{b}\end{array}$ & $\begin{array}{c}\text { Clathrate }(5.6 \mathrm{~K}) \\
\bar{v}\left(\mathrm{~cm}^{-1}\right)^{b}\end{array}$ \\
\hline \multirow{5}{*}{$3 v_{4}$} & 3826.1 & 3837.0 \\
\hline & 3843.2 & 3842.0 \\
\hline & 3847.9 & 3847.0 \\
\hline & 3849.8 & (3855.2) \\
\hline & 3851.8 & \\
\hline \multirow[t]{2}{*}{$v_{2}+2 v_{4}$} & 4113.6 & 4119.7 \\
\hline & 4120.0 & \\
\hline \multirow[t]{6}{*}{$v_{1}+v_{4}$} & 4201.8 & 4188.2 \\
\hline & 4206.3 & 4192.8 \\
\hline & & 4197.4 \\
\hline & & $4200.9 \mathrm{sh}$ \\
\hline & & 4206.8 \\
\hline & & 4210.8 \\
\hline \multirow[t]{8}{*}{$v_{3}+v_{4}$} & 4283.1 & 4283.9 \\
\hline & 4300.4 & 4290.3 \\
\hline & 4308.0 & $4296.1 \mathrm{sh}$ \\
\hline & 4314.5 & 4302.5 \\
\hline & 4323.7 & 4311.9 \\
\hline & 4327.6 & $4317.9 \mathrm{sh}$ \\
\hline & & 4323.9 \\
\hline & & $4332.0 \mathrm{br}$ \\
\hline \multirow[t]{5}{*}{$v_{2}+v_{3}$} & 4527.5 & 4506.6 \\
\hline & 4537.4 & 4515.7 \\
\hline & 4546.5 & 4525.0 \\
\hline & & 4533.3 \\
\hline & & 4540.0 \\
\hline \multirow[t]{3}{*}{$v_{3}+2 v_{4}$} & 5565.5 & 5545.1 \\
\hline & 5573.6 & 5553.2 \\
\hline & 5586.1 & 5560.5 \\
\hline \multirow[t]{3}{*}{$2 v_{2}+2 v_{4}$} & 5594.9 & 5594.3 \\
\hline & 5611.5 & 5605.4 \\
\hline & 5631.2 & \\
\hline \multirow[t]{2}{*}{$v_{2}+v_{3}+v_{4}$} & 5800.3 & 5782.9 \\
\hline & 5807.1 & 5792.0 \\
\hline \multirow[t]{5}{*}{$2 v_{3}$} & 5986.1 & 5959.7 \\
\hline & 6000.3 & 5968.6 \\
\hline & & 5977.4 \\
\hline & & (5988.9) \\
\hline & & (5997.3) \\
\hline
\end{tabular}

Notes. ${ }^{(a)}$ This work, based on the analysis presented in Baciocco et al. (1987a), Khanna \& Ngoh (1990), Calvani et al. (1992); ${ }^{(b)}$ the position typical accuracy is estimated to be better than $0.5 \mathrm{~cm}^{-1}$; lines in parenthesis are tentative detections

from directly assign a given cage environment for the observed transitions. The large to small cage favoured occupancy expected $(2 \mathrm{~S}$ versus $6 \mathrm{M})$ allowed us to tentatively attribute the strongest transitions in the $v_{3} \mathrm{CH}$-stretching mode region to methane in the large cages (Dartois \& Deboffle 2008). However, to confirm these assignments and improve the sensitivity to small cage transitions, we thus forced the clathrate hydrate small cages occupancy by using mixed clathrate properties. The relative variation of methane infrared active transitions in $\mathrm{CH}_{4} / \mathrm{CF}_{4}$ mixed clathrate as compared to the pure one, shown in Figs. 7 and 8 for $\mathrm{CH}$ fundamental modes and first combinations, respectively, confirm the identifications proposed. For $\mathrm{CH}$ stretching modes, when the absorptions are normalized to the sharpest transitions (indicated by an arrow labeled $\mathrm{N}$ for "normalized"), the two broader ones increase with $\mathrm{CF}_{4}$ content. The sharper transitions can thus be attributed to $\mathrm{CH}_{4}$ molecules in large cages, whose proportion diminishes with $\mathrm{CF}_{4}$ increase, the others to small cage entrapped methane transitions. A relative cage occupancy
Table 2. Relative NIR clathrate hydrate and methane ice integrated intensities ${ }^{a}$.

\begin{tabular}{lcc}
\hline \hline Dominant transitions & Clathrate $(5.6 \mathrm{~K})$ & Methane ice $^{b}$ \\
\hline $3 v_{4}$ & 0.063 & $\sim 0.054^{c}$ \\
$v_{2}+2 v_{4}$ & 0.010 & 0.013 \\
$v_{1}+v_{4}$ & 0.511 & 0.524 \\
$v_{3}+v_{4}$ & $1.0^{a}$ & $1.0^{a}$ \\
$v_{2}+v_{3}$ & 0.099 & 0.095 \\
$v_{3}+2 v_{4}$ & 0.014 & 0.014 \\
$2 v_{2}+2 v_{4}$ & 0.004 & $\sim 0.004^{d}$ \\
$v_{2}+v_{3}+v_{4}$ & 0.028 & 0.029 \\
$2 v_{3}$ & 0.042 & 0.061 \\
\hline
\end{tabular}

Notes. ${ }^{(a)}$ Normalised to the $v_{3}+v_{4}$ transitions; ${ }^{(b)}$ Brunetto et al. (2008) at $16 \mathrm{~K} ;{ }^{(c)}$ intensity before normalisation estimated from the ratio of absorption coefficient of $\left(v_{1}+v_{4}\right) / 3 v_{4}$ from Quirico \& Schmitt (1997) at $21 \mathrm{~K}$, assuming the same line width for both transitions; ${ }^{(d)}$ integrated intensity before normalisation estimated from the spectrum of the blended $\left(2 v_{2}+2 v_{4}\right) /\left(v_{3}+2 v_{4}\right)$ lines from Fig. $2 b$ in Quirico \& Schmitt (1997) at $21 \mathrm{~K}$.

Table 3. Calculated cages occupancy equilibrium.

\begin{tabular}{ccccc}
\hline \hline $\begin{array}{c}\mathrm{CH}_{4} \\
\text { (mol. ratio \%) }\end{array}$ & $\begin{array}{c}\text { Temperature }^{a} \\
(\mathrm{~K})\end{array}$ & $\begin{array}{c}\text { Pressure }^{a} \\
(\text { Bar })\end{array}$ & $\begin{array}{c}\text { Obs. }^{b} \\
\text { L/S }\end{array}$ & $\begin{array}{c}\text { Pred. }^{b} \\
\text { L/S }\end{array}$ \\
\hline 5 & 250 & 40 & $0.46 \pm 0.2$ & 0.45 \\
15 & 255 & 50 & $0.9 \pm 0.16$ & 0.86 \\
50 & 240 & 60 & $1.61 \pm 0.29$ & 1.78 \\
100 & 255 & 40 & 3.00 & 3.00 \\
\hline
\end{tabular}

Notes. ${ }^{(a)}$ Formation conditions; ${ }^{(b)}$ assuming and normalised to L/S $=3$ for the $100 \%$ methane clathrate hydrate

can be estimated from our spectra by integrating the peak area ratio and normalizing to the pure methane clathrate hydrate.

Combining $\mathrm{CH}$ stretch and combinations, we obtained the large to small occupancies given in Table 3, if a theoretical cage statistic of $\mathrm{L} / \mathrm{S}=3\left(100 \% \mathrm{CH}_{4}\right)$ is assumed and the absorption intrinsic intensities of the $\mathrm{CH}_{4}$ transition remains the same in all mixtures.

\subsection{Cages occupancy modelling}

In order to compare with our experimental results, we modelled the expected cage occupancies in the type I clathrate structure.

To model the cage occupancies, following the recent work of Ballard \& Sloan (2002), the guest fraction $\theta_{J, m}$ in the hydrate is given by the following equation:

$\theta_{J=\left\{\mathrm{CH}_{4} ; \mathrm{CF}_{4}\right\}, m=\{\mathrm{I} ; \mathrm{II}\}}=\frac{C_{J, m} f_{J, m}}{1+\sum_{k} C_{k, m} f_{k, m}}$

where $J$ stands for $\mathrm{CH}_{4}$ or $\mathrm{CF}_{4}$ in our case, and $m$ for either type I or II clathrate cavity. $f_{J, m}$ is the fugacity of guest $J$ in the cage $m . C_{J, m}$ is the associated Langmuir constant of guest $J$ in cavity $m$, and is given by

$C_{J, m}=\frac{4 \pi}{k T} \int_{0}^{R_{1}-a_{J}} \exp \left[-\frac{\sum_{n} \omega_{J, n}(r)}{k T}\right] r^{2} \mathrm{~d} r$.

The Langmuir constant describes the potential interaction between the encaged guest molecule and the water molecules forming the cages. In the Ballard \& Sloan model, the potential is evaluated up to the first "shell" $\left(\mathrm{R}_{1}\right)$ by summing over the distinct water molecules positions in the cages $\left(\sum_{n}\right)$, assuming a 


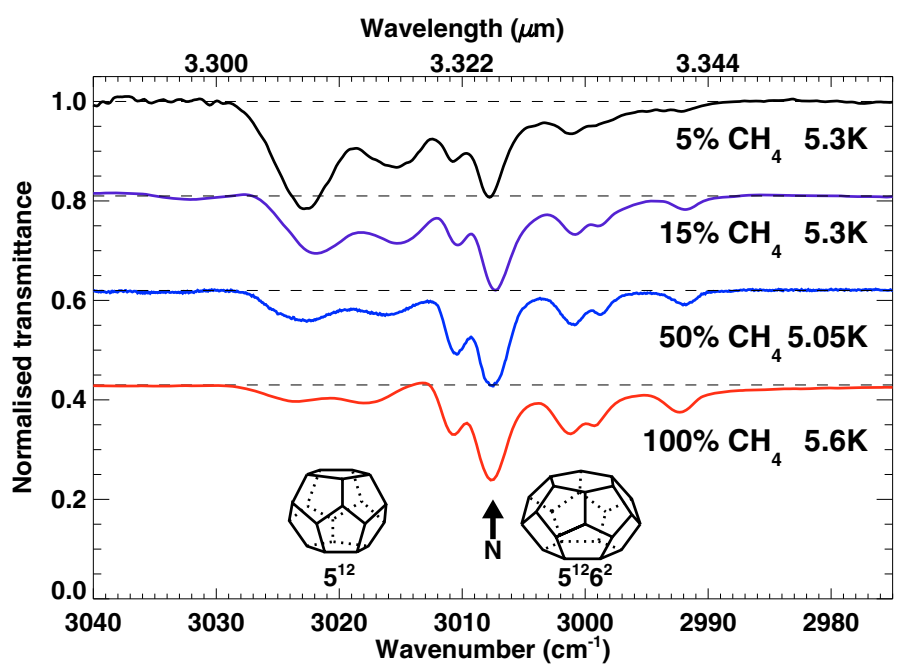

Fig. 7. Stretching-mode FTIR spectrum of the methane clathrate hydrate as recorded at $7 \mathrm{~K}$, compared to methane/carbon tetrafluoride mixed clathrates at $5.3 \mathrm{~K}$ starting with a gas-phase-ratio of $5 \%$ and $15 \%$, and $5.05 \mathrm{~K}$ with a gas-phase-ratio of $50 \%$, respectively. The transmittance of each spectrum was normalised to the $100 \% \mathrm{CH}_{4}$ clathrate $\sim 3008 \mathrm{~cm}^{-1}$ line for comparison. The arrow indicates the line used for the normalisation (labeled $\mathrm{N}$ ).

spherically symmetric cage and the guest Kihara spherical core potential. It gives rise to the interacting cell potential given by

$$
\begin{aligned}
& \omega_{J, m}(r)= \\
& 2 \epsilon_{J} z_{m}\left[\frac{\sigma_{J}^{12}}{R_{m}^{11} r}\left(\delta_{J, m}^{10}+\frac{a_{J}}{R_{m}} \delta_{J, m}^{10}\right)-\frac{\sigma_{J}^{6}}{R_{m}^{5} r}\left(\delta_{J, m}^{4}+\frac{a_{J}}{R_{m}} \delta_{J, m}^{5}\right)\right]
\end{aligned}
$$

with

$\delta_{J, m}^{N}=\frac{1}{N}\left[\left(1-\frac{r}{R_{m}}-\frac{a_{J}}{R_{m}}\right)^{-N}-\left(1+\frac{r}{R_{m}}-\frac{a_{J}}{R_{m}}\right)^{-N}\right]$.

We adopt the Mooijer-van den Heuvel et al. (2002) Kihara parameters for $\mathrm{CF}_{4}\left(\sigma_{\mathrm{CF}_{4}}=3.17 \AA, a_{\mathrm{CF}_{4}}=0.674 \AA, \epsilon_{\mathrm{CF}_{4}} / k=\right.$ $153.9 \mathrm{~K})$ and $\mathrm{CH} 4\left(\sigma_{\mathrm{CH}_{4}}=3.165 \AA, a_{\mathrm{CH}_{4}}=0.3834 \AA, \epsilon_{\mathrm{CH}_{4}} / k=\right.$ $154.54 \mathrm{~K}$ ) to model the cage occupancies.

In the type I structure limit, our findings presented in Table 3 agree relatively well with the model, which further confirms the assignments. The assignment is now secured and can also be performed in the near-infrared as shown in Fig. 8, normalising the spectra to the $v_{1}+v_{4}$ large cage transitions.

\subsection{Astrophysical implications}

Some discussion was initiated in a letter dedicated to the $\mathrm{CH}$ stretching mode of type I methane clathrate (Dartois \& Deboffle 2008). We also previously investigated the carbon dioxide type I clathrate FTIR spectrum in the near-infrared (Dartois et al. 2009) to complete mid-infrared previous measurements, and the readers are invited to include the spectroscopic implications discussed there, which remain valid.

Methane-ice transitions have been remotely detected in many objects of the solar system. Among objects linked to the trans-Neptunian region and Kuiper belt, $\mathrm{CH}_{4}$ is observed in Pluto (Cruikshank et al. 1976; Metz 1976), Makemake (2005, $\mathrm{FY}_{9}$; Barkume et al. 2005; Licandro et al. 2006), Eris (2003, $\mathrm{UB}_{313}$; Dumas et al. 2007), Sedna (e.g. Barucci et al. 2005; Trujillo et al. 2005), Quaoar (Schaller \& Brown 2007), Triton

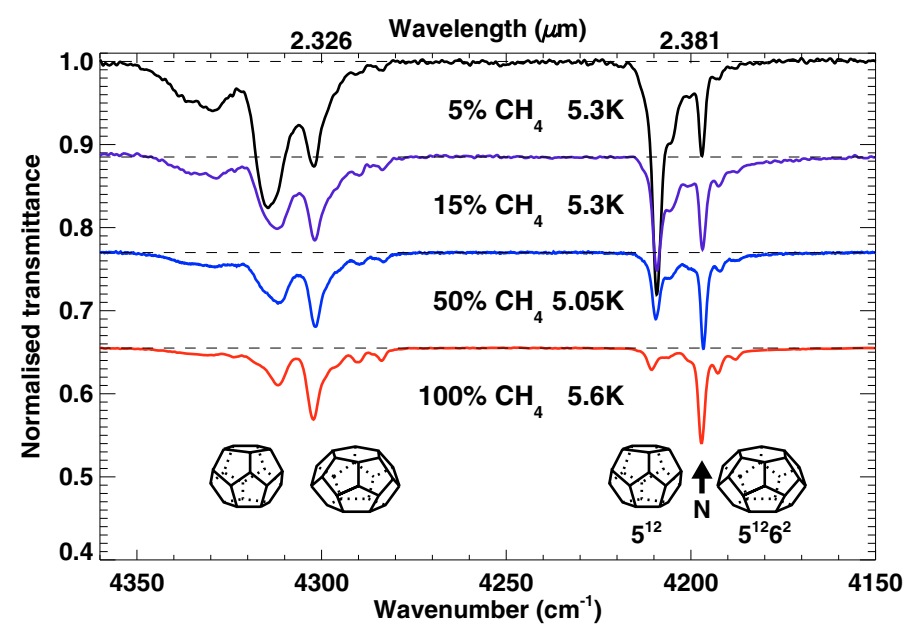

Fig. 8. Same set of spectra as in Fig. 7 for mixed methane/carbon tetrafluoride clathrate hydrates in the $v_{1}+v_{4}$ and $v_{3}+v_{4}$ combination mode absorption range. The arrow indicates the line used for the normalisation (labeled $\mathrm{N}$ ).

(Cruikshank \& Apt 1984). When accessible to high resolution spectra, however, the position of the methane lines does not correspond to the methane clathrate reported in this article. For Pluto it is blue-shifted and shown to be embedded into the also detected $\beta-\mathrm{N}_{2}$ ice (e.g. Douté et al. 1999; Grundy \& Buie 2001), whereas the shift for Makemake (Brown et al. 2007) is intermediate between Pluto's and pure methane-ice absorptions. A similar $\beta$-nitrogen matrix-shift is observed for Triton (e.g. Cruikshank et al. 1993; Quirico et al. 1999; Grundy \&Young 2004). In addition, in many of these objects the water-ice does not dominate the inventory of surface-observed species. In their core, water-ice is probably present, and if the surface has been replenished in methane recently by outgassing from the interior, a very strong driving force must be at work if these species are trapped as clathrate hydrates.

Closer to Earth, methane was observed recently in the atmosphere of Mars (e.g. Formisano et al. 2004, and references therein), although the methane abundance is still much debated. Some models invoke the possibility of a methane clathrate source (e.g. Chastain \& Chevrier 2007; Chassefiere 2009). If that existed, it would escape direct remote infrared detection, because it must lie well under the surface to be stable on a geological time scale. The peculiar molecular and rare gas abundance pattern observed in giant planets and the late release of volatiles from comets has also been associated to possible modifications induced by clathrate hydrates trapping in the proto-solar nebula (e.g. Lunine \& Stevenson1985; Gautier et al. 2001; Iro et al. 2003; Hersant et al. 2008).

At least three aspects can be explored, which touch the numerous modellings or observations of clathrates in astrophysics. The first is thermodynamics, the other two are kinetics and spectroscopy (both often neglected in constraining the astrophysical problem). Thermodynamics provides, in particular, phase diagrams with stability curves, a necessary condition to meet for the clathrates' existence, but leading to very high upper limits in models when formation kinetic is neglected. Kinetics may be the limiting factor in some astrophysical bodies. Assessing a possible presence of clathrate hydrates based on thermodynamics is a first step, and if efforts must be undertaken to measure their thermodynamic properties at astrophysical temperatures, clathrate hydrates must be concomitantly searched for observationally by spectroscopic means. The spectra indicate that at temperatures 
higher than $80 \mathrm{~K}$, rotational lines are broadened (as compared to the sharper very low temperature transitions), a temperature closer to most of the outer solar system water-dominant ice surfaces (100-120 K Jovian and Saturnian). The shift and degeneracy lift induced by the cages should still be observable through remote sensing. In the context of outer solar system objects, their intrinsic lower temperatures increase the chance to detect clathrates (both from spectroscopy and thermodynamics) and a focus on e.g. the TNOs richest in water-ice is desirable.

\section{Conclusion}

We conducted pure methane and mixed methane-carbon tetrafluoride clathrate hydrate formation in a medium pressure closedcell and analysed their FTIR spectra to explore a broad range of astrophysical spectroscopic identification. By varying the cage occupancies, which is related to the mixture ratios, we disentangled the line assignments of the different cages for methane in type I clathrate hydrate stretching modes, combination and overtones, and confirmed our previous analysis in the stretching mode range. The small and large cage type I methane clathrate transitions identification was performed for evident spectral simplification reasons at the lowest temperature reached in the experiments, which is only relevant to interstellar dense clouds environments, but drives the identification for the higher temperatures $(77 \mathrm{~K})$ clathrates spectra recorded closer to solar system objects conditions. Because the small cage is common to the two main clathrates structures (type I and II clathrates forms), it provides a way to unequivocally identify the presence of methane encaged in such a structure at low temperatures.

An outcome for observational astrophysics of this work is that the wavelength range covered (1.65 to $3.4 \mu \mathrm{m})$ by our dataset allows us to probe transitions accessible both to planetary (combinations and overtones) reflectance and interstellar medium ( $\mathrm{CH}$ stretching mode) transmittance remote spectroscopy, taking into account the distinct opacity regimes. Up to now, the astrophysically observed methane is involved in other interactions, sometimes less stable from the thermodynamics point of view than clathrates. We are currently experimentally investigating the formation of the type II methane clathrate structure for completeness of the analysis. The work presented here provides a spectroscopic dictionary to allow either clathrate detection or stringent upper limit non-detections. Immediately following, we are building low temperature laboratory kinetic experiments, for which spectroscopy is an invaluable constraint.

Acknowledgements. We acknowledge fruitful discussions and/or technical support from A. Arondel, A. Chardin, N. Coron, B. Crane, D. Deboffle, C. Dumesnil, J.-J. Fourmond, L. d'Hendecourt, F. Langlet, T. Redon, G. Renoux as well as A. Peter for a thorough reading of the article.

\section{References}

Baciocco, G., Calvani, P., \& Cunsolo, S. 1987a, J. Chem. Phys., 87, 1913 Baciocco, G., Calvani, P., \& Cunsolo, S. 1987b, International Journal of Infrared and Millimeter Waves, 8, 923

Ballard, A. L., \& Sloan, E. D. 2002, Fluid Phase Equilibria, 194, 371

Barkume, K. M., Brown, M. E., \& Schaller, E. L. 2005, BAAS, 37, 738

Barucci, M. A., Cruikshank, D. P., Dotto, E., et al. 2005, A\&A, 439, L1

Brown, M. E., Barkume, K. M., Blake, G. A., et al. 2007, AJ, 133, 284

Brunetto, R., Caniglia, G., Baratta, G. A., \& Palumbo M. E. 2008, ApJ, 686, 1480

Calvani, P., Cunsolo, S., Lupi, S., \& Nucara, A. 1992, J. Chem. Phys., 96, 7372

Chapados, C., \& Cabana, A. 1972, Can. J. Chem., 50, 3521

Chastain, B. K., \& Chevrier, V. 2007, Planet. Space Sci., 55, 1246

Chatti, I., Delahaye, A., Fournaison, L., \& Petitet, J.-P. 2005, Energy Conversion and Managment, 46, 1333

Cruikshank, D. P., \& Apt, J. 1984, Icarus, 58, 306

Cruikshank, D. P., Pilcher, C. B., \& Morrison, D. 1976, Science, 194, 835

Cruikshank, D. P., Roush, T. L., Owen, T. C., et al. 1993, Science, 261, 742

Dartois, E., \& Deboffle, D. 2008, A\&A, 490, L19

Dartois, E., \& Schmitt, B. 2009, A\&A, 504, 869

Delsemme, A. H., \& Swings, P. 1952, Ann. Astrophys., 15, 1

Douté, S., Schmitt, B., Quirico, E., et al. 1999, Icarus, 142, 421

Dumas, C., Merlin, F., Barucci, M. A., et al. 2007, A\&A, 471, 331

Faraday, M. 1823, On Hydrate of Chlorine, Quartly J. Sci., 15, 71

Formisano, V., Atreya, S., Encrenaz, T., Ignatiev, N., \& Giuranna, M. 2004, Science, 306, 1758

Gautier, D., Hersant, F., Mousis, O., \& Lunine, J. I. 2001, ApJ, 550, L227

Grundy, W. M., \& Buie, M. W. 2001, Icarus, 153, 248

Grundy, W. M., \& Young, L. A. 2004, Icarus, 172, 455

Grundy, W. M., Schmitt, B., \& Quirico, E. 2002, Icarus, 155, 486

Hersant, F., Gautier, D., \& Lunine, J. I. 2004, Planet. Space Sci., 52, 623

Hersant, F., Gautier, D., Tobie, G., \& Lunine, J. I. 2008, Planet. Space Sci., 56, 1103

Iro, N., Gautier, D., Hersant, F., Bockelée-Morvan, D., \& Lunine, J. I. 2003, Icarus, 161, 511

Khanna, R. 1990, Spectrochim. Acta Part A: Mol. Spectrosc., 46, 1057

Kobashi, K., Okada, K., \& Yamamoto, T. 1977, J. Chem. Phys., 66, 5568

Kuhs, W. F., \& Klapproth, A. 2000, Second International Conference on Mars Polar Science and Exploration, 100

Kunita, Y., Makino, T., Sugahara, T., \& Ohgaki, K. 2007, Fluid Phase Equilibria, 251,145

Licandro, J., Pinilla-Alonso, N., Pedani, M., et al. 2006, A\&A, 445, L35

Lunine, J. I., \& Stevenson, D. J. 1985, ApJS, 58, 493

Metz. W. D. 1976, Science, 192, 362

Miller, S. L. 1961, Proceedings of the National Academy of Science, 47, 1798

Mooijer-van den Heuvel, M. M., Peters C. J., \& de Swaan Arons, J. 2002, Fluid Phase Equilibria, 172, 73

Mooijer-van den Heuvel, M. M., Sawirjo, N. M., \& Peters C. J. 2006, Fluid Phase Equilibria, 241, 124

Mousis, O., \& Schmitt, B. 2008, ApJ, 677, L67

Quirico, E., \& Schmitt, B. 1997, Icarus, 127, 357

Quirico, E., Doute, S., Schmitt, B., et al. 1999, Icarus, 139, 159

Schaller, E. L., \& Brown, M. E. 2007, ApJ, 670, L49

Schmitt, B., Mulato, L., \& Douté, S. 2003, Third International Conference on Mars Polar Science and Exploration, 8073

Sill, G. T., \& Wilkening, L. 1978, Icarus, 33, 13

Trujillo, C. A., Brown, M. E., Rabinowitz, D. L., \& Geballe, T. R. 2005, ApJ, 627,1057

Vogt, G. J., \& Pitzer, K. S. 1976, J. Chem. Thermodynam., 8, 1011 